\title{
Prospects for high temperature ferromagnetism in (Ga,Mn)As semiconductors
}

\author{
T. Jungwirth, ${ }^{1,2}$ K. Y. Wang, ${ }^{2}$ J. Mašek, ${ }^{3}$ K. W. Edmonds, ${ }^{2}$ Jürgen König, ${ }^{4}$ Jairo Sinova, ${ }^{5}$ M. Polini, ${ }^{6}$ N. A. Goncharuk, \\ A. H. MacDonald, ${ }^{7}$ M. Sawicki, ${ }^{8}$ A. W. Rushforth, ${ }^{2}$ R. P. Campion, ${ }^{2}$ L. X. Zhao, ${ }^{2}$ C. T. Foxon, ${ }^{2}$ and B. L. Gallagher ${ }^{2}$ \\ ${ }^{1}$ Institute of Physics ASCR, Cukrovarnická 10, 16253 Praha 6, Czech Republic \\ ${ }^{2}$ School of Physics and Astronomy, University of Nottingham, Nottingham NG7 2RD, United Kingdom \\ ${ }^{3}$ Institute of Physics ASCR, Na Slovance 2, 18221 Praha 8, Czech Republic \\ ${ }^{4}$ Institut für Theoretische Physik III, Ruhr-Universität Bochum, 44780 Bochum, Germany \\ ${ }^{5}$ Department of Physics, Texas A\&M University, College Station, Texas 77843-4242, USA \\ ${ }^{6}$ NEST-INFM and Scuola Normale Superiore, I-56126 Pisa, Italy \\ ${ }^{7}$ Department of Physics, University of Texas at Austin, Austin, Texas 78712-1081, USA \\ ${ }^{8}$ Institute of Physics, Polish Academy of Sciences, 02668 Warszawa, Poland
}

(Received 9 May 2005; revised manuscript received 21 July 2005; published 13 October 2005)

\begin{abstract}
We report on a comprehensive combined experimental and theoretical study of Curie temperature trends in $(\mathrm{Ga}, \mathrm{Mn})$ As ferromagnetic semiconductors. Broad agreement between theoretical expectations and measured data allows us to conclude that $T_{c}$ in high-quality metallic samples increases linearly with the number of uncompensated local moments on $\mathrm{Mn}_{\mathrm{Ga}}$ acceptors, with no sign of saturation. Room temperature ferromagnetism is expected for a $10 \%$ concentration of these local moments. Our magnetotransport and magnetization data are consistent with the picture in which $\mathrm{Mn}$ impurities incorporated during growth at interstitial $\mathrm{Mn}_{\mathrm{I}}$ positions act as double-donors and compensate neighboring $\mathrm{Mn}_{\mathrm{Ga}}$ local moments because of strong nearneighbor $\mathrm{Mn}_{\mathrm{Ga}}-\mathrm{Mn}_{\mathrm{I}}$ antiferromagnetic coupling. These defects can be efficiently removed by post-growth annealing. Our analysis suggests that there is no fundamental obstacle to substitutional $\mathrm{Mn}_{\mathrm{Ga}}$ doping in high-quality materials beyond our current maximum level of $6.8 \%$, although this achievement will require further advances in growth condition control. Modest charge compensation does not limit the maximum Curie temperature possible in ferromagnetic semiconductors based on (Ga,Mn)As.
\end{abstract}

DOI: 10.1103/PhysRevB.72.165204

PACS number(s): 75.50.Pp, 75.30.Gw, 73.61.Ey

\section{INTRODUCTION}

After some frustration in the community caused by the difficulties encountered in overcoming the apparent Curie temperature limit in $(\mathrm{Ga}, \mathrm{Mn})$ As of $T_{c}=110 \mathrm{~K},{ }^{1-4}$ the record transition temperature has been steadily increasing over the last two years. ${ }^{5-9}$ The maximum $T_{c}=173 \mathrm{~K}$ reported ${ }^{9}$ to date is likely another short-lived record in bulk (Ga,Mn)As ferromagnets. It is now established that the success has been made possible by the technological progress in controlling crystallographic quality of the materials, namely, in reducing the number of unintentional charge and moment compensating defects through optimized growth and post-growth annealing procedures. ${ }^{3-10}$ Experiments also suggest that the general picture of ferromagnetism that applies to these metallic $(\mathrm{Ga}, \mathrm{Mn})$ As systems is the one in which magnetic coupling between local Mn moments is mediated by delocalized holes in the $(\mathrm{Ga}, \mathrm{Mn})$ As valence band. The fact that the mechanism does not imply a fundamental $T_{c}$ limit below room temperature motivates a detailed analysis of our understanding of the $T_{c}$ trends in currently available high quality metallic materials with $\mathrm{Mn}$ doping ranging from approximately $2 \%$ to $9 \%$.

Curie temperatures in metallic (Ga,Mn)As have been studied theoretically starting from semiphenomenological ${ }^{11-15}$ and microscopic models ${ }^{16-21}$ of the electronic structure. The former approach asserts a localized character of the five $\mathrm{Mn}_{\mathrm{Ga}} d$ orbitals forming a moment $S$ $=5 / 2$ and describes hole states in the valence band using the Kohn-Luttinger parameterization for GaAs (Ref. 22) and a single constant $J_{p d}$ which characterizes the exchange interaction between $\mathrm{Mn}_{\mathrm{Ga}}$ and hole spins. The exchange interaction follows from hybridization between Mn $d$ orbitals and valence band $p$ orbitals. The semiphenomenological Hamiltonian implicitly assumes that a canonical transformation has been performed which eliminated the hybridization. ${ }^{21}$ In this approach the hybridization is implicitly assumed to be weak in several different ways, and the canonical transformation ignored in representing observables. Although this approach is consistent, it should be realized that the localized $d$ orbitals in the phenomenological Hamiltonian are in reality hybridized with the valence band.

The advantage of the semiphenomenological approach is that it uses the experimental value ${ }^{23,24}$ for $J_{p d}$ $=54 \pm 9 \mathrm{meV} \mathrm{nm}^{3}$, i.e., it correctly captures the strength of the magnetic interaction that has been established to play the key role in ferromagnetism in $(\mathrm{Ga}, \mathrm{Mn}) \mathrm{As}$. The model also accounts for strong spin-orbit interaction present in the host valence band which splits the three $p$ bands into a heavyhole, light-hole, and a split-off band with different dispersions. The spin-orbit coupling is not only responsible for a number of distinct magnetic ${ }^{25-28}$ and magneto-transport ${ }^{29-32}$ properties of $(\mathrm{Ga}, \mathrm{Mn})$ As ferromagnets but the resulting complexity of the valence band was shown ${ }^{14,33}$ to play also an important role in suppressing magnetization fluctuation effects and, therefore, stabilizing the ferromagnetic state itself. On the other hand, describing the potentially complex behavior of $\mathrm{Mn}_{\mathrm{Ga}}$ in GaAs by a single parameter may oversimplify the problem. The calculations omit, for example, the contribution of direct antiferromagnetic superexchange to the cou- 
pling of near-neighbor Mn pairs, and the whole model inevitably breaks down if valence fluctuations of $\mathrm{Mn}_{\mathrm{Ga}} d$ electrons become strong.

Microscopic theories, whether based on the tight-bindingapproximation (TBA) parameterization of energies and overlaps of valence orbitals in the lattice ${ }^{20,21}$ or on the $a b$ initio local-density-approximation (LDA) schemes, ${ }^{16-19}$ make no assumption upon the character of $\mathrm{Mn}_{\mathrm{Ga}}$ impurities in GaAs and their magnetic coupling. They are therefore useful for studying material trends in $T_{c}$ as a function of Mn doping or density and position in the lattice of other intentional or unintentional impurities present in real systems. ${ }^{34}$ Because spin-orbit interactions add to the numerical complexity of calculations that are already challenging, they have normally been neglected. Another shortcoming of the $a b$ initio approaches is the incomplete elimination of self-interaction effects which leads to smaller relative displacement of the Mn $d$ levels and the top of the valence band. This results in an overestimated strength of the $p-d$ exchange as compared to experiment.

Within the mean-field approximation, which considers thermodynamics of an isolated Mn moment in an effective field and neglects correlated $\mathrm{Mn}-\mathrm{Mn}$ fluctuations, microscopic calculations ${ }^{18}$ typically yield larger $T_{c}$ 's than the semiphenomenological models ${ }^{13,14}$ that use the experimental $J_{p d}$ value. Stronger $p$ - $d$ exchange in the microscopic theories leads, however, also to a larger suppression of the Curie temperature due to fluctuation effects, especially so in highly doped systems. ${ }^{18}$ (A closer agreement in the character of the $T_{c}$ vs Mn-doping curves, calculated within the two formalisms, is obtained when the deficiencies of density-functional theories are partly eliminated by introducing a correlation energy constant within the LDA+U schemes. ${ }^{18}$ ) Despite the above weaknesses of semiphenomenological and microscopic calculations, an overall, qualitatively consistent picture is clearly emerging from these complementary theoretical approaches that, as we discuss below, provides a useful framework for analyzing measured $T_{c}$ 's.

In experimental Curie temperature studies it is crucial to decouple intrinsic properties of $(\mathrm{Ga}, \mathrm{Mn}) \mathrm{As}$ ferromagnets from extrinsic, effects due to the presence of unintentional impurities. Arsenic antisites $\left(\mathrm{As}_{\mathrm{Ga}}\right)$ and interstitial manganese $\left(\mathrm{Mn}_{\mathrm{I}}\right)$ represent two major sources of charge compensation in $(\mathrm{Ga}, \mathrm{Mn})$ As grown by low-temperature molecular beam epitaxy (LT-MBE), both acting as double-donors. ${ }^{35,36}$ A $\mathrm{Mn}_{\mathrm{I}}$ cation when attracted to a $\mathrm{Mn}_{\mathrm{Ga}}$ anion compensates also the $\mathrm{Mn}_{\mathrm{Ga}}$ local moment as the two species are expected to couple antiferromagnetically $8,37,38$ due to superexchange over the whole range from strong to weak charge compensation.

The $\mathrm{As}_{\mathrm{Ga}}$ antisites are stable ${ }^{39}$ up to $\sim 450{ }^{\circ} \mathrm{C}$, which is well above the transition temperature from a uniform diluted magnetic semiconductor to a multiphase structure with metallic MnAs and other precipitates. Therefore, the number of $\mathrm{As}_{\mathrm{Ga}}$ defects has to be minimized already during the LTMBE growth by precisely controlling the stoichiometry of deposited epilayers. ${ }^{40}$ The $\mathrm{Mn}_{\mathrm{I}}$ impurity concentration can be significant in as-grown structures. These defects are, however, much more mobile than the As antisites. During annealing at temperatures close to the MBE growth temperature $\sim 200{ }^{\circ} \mathrm{C}$ they out-diffuse and are passivated at the epilayer surface. ${ }^{8}$

In this paper we have collected data for a set of samples that show very weak charge and moment compensation after annealing, i.e., a negligible number of $\mathrm{As}_{\mathrm{Ga}}$, which allows us to determine experimentally $T_{c}$ trends related to intrinsic properties of (Ga,Mn)As ferromagnets. We also point out that conclusions made here are based on magnetic and transport measurements in 16 different $(\mathrm{Ga}, \mathrm{Mn})$ As materials (8 as-grown and 8 annealed) which we fabricated and analyzed using consistent experimental procedures and which, therefore, represent a unique comprehensive set of measurements. A direct quantitative comparison with results obtained by other groups $1,2,4,6,7,10$ is hindered by incompatabilities in the employed growth and characterization techniques. Nevertheless, the behavior of materials synthetized in different laboratories clearly follows similar patterns for Mn doping up to $\sim 5 \%$. In samples with higher Mn content the levels and nature of compensating defects can vary more strongly from material to material depending on the details of the growth and post-growth annealing procedures. This may explain large differences in conductivities and Curie temperatures reported by different groups in highly doped (Ga,Mn)As materials.

The paper is organized as follows: In Sec. II we start with the semiphenomenological mean-field approximation (Sec. II A) to set up a scale of expected Curie temperatures in the material, assuming homogeneous distribution of $\mathrm{Mn}_{\mathrm{Ga}}$ ions (the virtual-crystal approximation). We then discuss various physically distinct effects that are not captured by this picture. In Sec. II B we evaluate the Stoner enhancement of the Curie temperature due to hole-hole exchange interaction. Suppression of $T_{c}$ due to antiferromagnetic superexchange contribution to the near-neighbor $\mathrm{Mn}_{\mathrm{Ga}}-\mathrm{Mn}_{\mathrm{Ga}}$ coupling in highly compensated samples ${ }^{41}$ is illustrated in Sec. II C. In this section we discuss also effects on $T_{c}$ arising from the discreteness of random $\mathrm{Mn}_{\mathrm{Ga}}$ positions in the lattice that becomes important in the opposite regime, i.e., in systems with low charge compensation or co-doped with additional nonmagnetic acceptors. Effects beyond the mean-field approximation, namely the disappearance of the ferromagnetic longrange order due to collective $\mathrm{Mn}_{\mathrm{Ga}}$ moments fluctuations are discussed in Sec. II D. Since the $\mathrm{Mn}_{\mathrm{Ga}}, \mathrm{Mn}_{\mathrm{I}}$, and hole densities represent key parameters in the discussion of measured Curie temperatures, we present in Sec. II E theoretical predictions for equilibrium partial concentrations of substitutional $\mathrm{Mn}_{\mathrm{Ga}}$ and interstitial $\mathrm{Mn}_{\mathrm{I}}$ impurities in as-grown samples, and in Sec. II F we estimate the accuracy of the Hall measurement of hole density in the polarized (Ga,Mn)As valence bands.

Measured $T_{c}$ and hole densities are presented in Sec. III A for a set of samples with different nominal Mn doping, before and after annealing. Motivated by the above theoretical analysis we determine in Sec. III B the partial density of $\mathrm{Mn}_{\mathrm{Ga}}$ and $\mathrm{Mn}_{\mathrm{I}}$, and the effective density of uncompensated $\mathrm{Mn}_{\mathrm{Ga}}$ local moments in our samples. The interpretation is based on total Mn-doping values, obtained from secondary ion mass spectroscopy (SIMS), and Hall measurements of the hole densities before and after annealing. Consistency of these results is checked by comparisons with independent 
magnetization measurements. In Sec. III C we present experimental $T_{c}$ dependencies on uncompensated $\mathrm{Mn}_{\mathrm{Ga}}$ moment and hole densities and compare the data with theory predictions. Technological issues related to the growth of (Ga,Mn)As epilayers with large Mn concentrations are discussed in Sec. IV. Our perspective on high-temperature ferromagnetism in $(\mathrm{Ga}, \mathrm{Mn}) \mathrm{As}$ semiconductors is summarized in Sec. V.

\section{THEORY}

\section{A. Mean-field virtual crystal approximation}

The description of ordered states in $(\mathrm{Ga}, \mathrm{Mn}) \mathrm{As}$ is greatly simplified by the virtual-crystal approximation in which the random $\mathrm{Mn}_{\mathrm{Ga}}$ distribution is replaced by a continuum with the same average local moment density and the role of other defects is neglected, apart from the potential hole or moment compensation. ${ }^{11,12,42,43}$ Microscopic TBA calculations showed $^{20}$ very little effect of positional disorder on the strength of magnetic couplings in $(\mathrm{Ga}, \mathrm{Mn})$ As epilayers with metallic conductivities of interest here, which partly justifies the virtual-crystal approach. Other detailed theoretical studies, corroborated by experimental data below, confirm the absence of any significant magnetic frustration in this ferromagnetic semiconductor associated with the random positions of $\mathrm{Mn}_{\mathrm{Ga}}$ moments in the lattice. ${ }^{44,45}$

In the mean-field approximation, ${ }^{11,12}$ each local $\mathrm{Mn}_{\mathrm{Ga}}$ moment is described by a Hamiltonian $\vec{S}_{I} \cdot \vec{H}_{M F}$ where $\vec{S}_{I}$ is the $\mathrm{Mn}_{\mathrm{Ga}}$ local spin operator, $\vec{H}_{M F}=J_{p d}\langle\vec{s}\rangle$, and $\langle\vec{s}\rangle$ is the mean spin density of the valence band holes. $H_{M F}$ is an effective field seen by the local moments due to spin polarization of the band holes, analogous to the nuclear Knight shift. Similarly $\vec{h}_{M F}=J_{p d} N_{\mathrm{Mn}}\langle\vec{S}\rangle$ is an effective magnetic field experienced by the valence band holes, where $\langle\vec{S}\rangle$ is the mean spin polarization of the $\mathrm{Mn}_{\mathrm{Ga}}$ local moments, and $N_{\mathrm{Mn}}=4 x / a_{\mathrm{lc}}^{3}$ is the $\mathrm{Mn}_{\mathrm{Ga}}$ density in $\mathrm{Ga}_{1-x} \mathrm{Mn}_{x}$ As with a lattice constant $a_{\mathrm{lc}}$. The dependence of $\langle\vec{S}\rangle$ on temperature and field $H_{M F}$ is given ${ }^{43}$ by the Brillouin function:

$$
\langle\vec{S}\rangle=\frac{\vec{H}_{M F}}{\left|H_{M F}\right|} S B_{s}\left(S\left|H_{M F}\right| / k_{B} T\right) .
$$

The Curie temperature is found by linearizing $H_{M F}$ and $B_{s}$ around $\langle\vec{S}\rangle=0$ :

$$
\begin{gathered}
\vec{H}_{M F} \approx J_{p d}^{2} N_{\mathrm{Mn}}\langle\vec{S}\rangle \chi_{f} \\
B_{s} \approx \frac{S+1}{3} \frac{S\left|H_{M F}\right|}{k_{B} T_{c}} .
\end{gathered}
$$

Here $\chi_{f}$ is the itinerant hole spin susceptibility given by

$$
\chi_{f}=\frac{d\langle s\rangle}{d h_{M F}}=-\frac{d^{2} e_{T}}{d h_{M F}^{2}}
$$

and $e_{T}$ is the total energy per volume of the holes. Equations (1) and (2) give

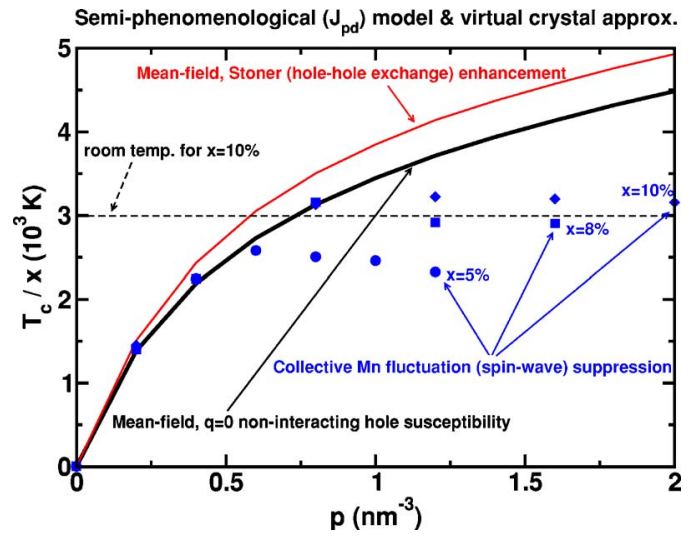

FIG. 1. (Color online) Ferromagnetic transition temperatures of (Ga,Mn)As calculated within the effective Hamiltonian and virtualcrystal approximation: mean-field (thick black line), Stoner enhancement of $T_{c}$ (thin line), spin-wave suppression of $T_{c}$ (closed diamonds, squares, and circles).

$$
k_{B} T_{c}=\frac{N_{\mathrm{Mn}} S(S+1)}{3} J_{p d}^{2} \chi_{f} .
$$

Qualitative implications of this $T_{c}$ Eq. (4) can be understood within a model itinerant hole system with a single spin-split band and an effective mass $m^{*}$. The kinetic energy contribution $e_{k}$ to the total energy of the band holes gives a susceptibility:

$$
\chi_{f, k}=-\frac{d^{2} e_{k}}{d h_{M F}^{2}}=\frac{m^{*} k_{F}}{4 \pi^{2} \hbar^{2}},
$$

where $k_{F}$ is the Fermi wave vector. Within this approximation $T_{c}$ is proportional to the $\mathrm{Mn}_{\mathrm{Ga}}$ density, to the hole Fermi wave vector, i.e., to $p^{1 / 3}$ where $p$ is the hole density, and to the hole effective mass $m^{*}$.

To obtain quantitative predictions for the critical temperature, it is necessary to evaluate the itinerant hole susceptibility using a realistic band Hamiltonian, $H=H_{K L}+\vec{s} \cdot \vec{h}_{M F}$, where $H_{K L}$ the six-band Kohn-Luttinger model of the GaAs host band ${ }^{22}$ and $\vec{s}$ is the hole spin operator. ${ }^{12,25,26}$ The results, represented by the solid black line in Fig. 1, are consistent with the qualitative analysis based on the parabolic band model, i.e., $T_{c}$ follows roughly the $\sim x p^{1 / 3}$ dependence. Based on these calculations, room temperature ferromagnetism in $(\mathrm{Ga}, \mathrm{Mn}) \mathrm{As}$ is expected for $10 \% \mathrm{Mn}_{\mathrm{Ga}}$ doping in weakly compensated samples.

\section{B. Stoner enhancement of $T_{c}$}

In highly doped (Ga,Mn)As epilayers the hole-hole correlation effects are weak and can be neglected. The exchange total energy $e_{x}$ adds a contribution to the hole spin susceptibility:

$$
\chi_{f, x}=-\frac{d^{2} e_{x}}{d h_{M F}^{2}}
$$

which for a single parabolic spin-split band reads, 


$$
\chi_{f, x}=\frac{e^{2}\left(m^{*}\right)^{2}}{4 \pi^{3} \varepsilon \hbar^{4}},
$$

where $\varepsilon$ is the dielectric constant of the host semiconductor. Equation (7) suggests a hole-density independent Stoner enhancement of $T_{c}$ proportional to $\mathrm{Mn}_{\mathrm{Ga}}$ concentration and $\left(m^{*}\right)^{2}$.

As in the noninteracting hole case discussed above, the detailed valence-band structure has to be accounted for to make quantitative estimates of the Stoner $T_{c}$ enhancement. The red line in Fig. 1 shows the Stoner $T_{c}$ enhancement calculated numerically from Eqs. (6). As expected, $T_{c}$ stays roughly proportional to $x p^{1 / 3}$ even if hole-hole exchange interactions are included, and the enhancement of the Curie temperature due to interactions is of the order $\sim 10-20 \%$.

\section{Discreteness of random $\mathrm{Mn}_{\mathrm{Ga}}$ positions, superexchange}

So far, the mean-field analysis of $T_{c}$ has neglected discreteness in random $\mathrm{Mn}_{\mathrm{Ga}}$ positions in the lattice and other magnetic coupling mechanisms; besides the kineticexchange, this includes the near-neighbor superexchange. The former point can be expected to affect $T_{c}$ at large hole densities, i.e., when the hole Fermi wavelength approaches inter-atomic distances. In the opposite limit of strongly compensated systems, where the overall magnitude of the holemediated exchange is weaker, antiferromagnetic superexchange can dominate the near-neighbor $\mathrm{Mn}_{\mathrm{Ga}}-\mathrm{Mn}_{\mathrm{Ga}}$ coupling, ${ }^{41}$ leading to a reduced Curie temperature. ${ }^{18,20}$ This type of magnetic interaction was ignored in the previous section. We emphasize that the phenomenological model cannot be applied consistently when nearest-neighbor interactions dominate, since it implicitly assumes that all length scales are longer than a lattice constant. We also note that net antiferromagnetic coupling of near-neighbor $\mathrm{Mn}_{\mathrm{Ga}}-\mathrm{Mn}_{\mathrm{Ga}}$ pairs is expected only in systems with large charge compensations. In weakly compensated (Ga,Mn)As the ferromagnetic contribution takes over. ${ }^{41,46}$

Besides the above effects of random Mn distribution, Mn positional disorder can directly modify the $p$ - $d$ interaction when the coherence of Bloch states becomes significantly disturbed. Microscopic theories, such as the TBA calculations ${ }^{20}$ presented in this section or approaches based on $a b$ initio LDA band structure, ${ }^{18}$ capture all these effects on an equal footing and can be used to estimate trends in mean-field $T_{c}$ beyond the virtual-crystal approximation. The theories do not assert any specific magnetic coupling mechanism from the outset. Instead, these follow from the microscopic description of the electronic structure of the doped crystal.

Within the coherent-potential approximation (CPA), disorder effects appear in the finite spectral width of hole quasiparticle states. Since realizations with near-neighbor $\mathrm{Mn}_{\mathrm{Ga}}$ ions are included within the disorder-averaged TBA/CPA with the proper statistical probability, short-range local moment interactions (such as superexchange) contribute to the final magnetic state.

The parametrization of our TBA Hamiltonian was chosen to provide the correct band gap for a pure GaAs crystal ${ }^{47}$ and

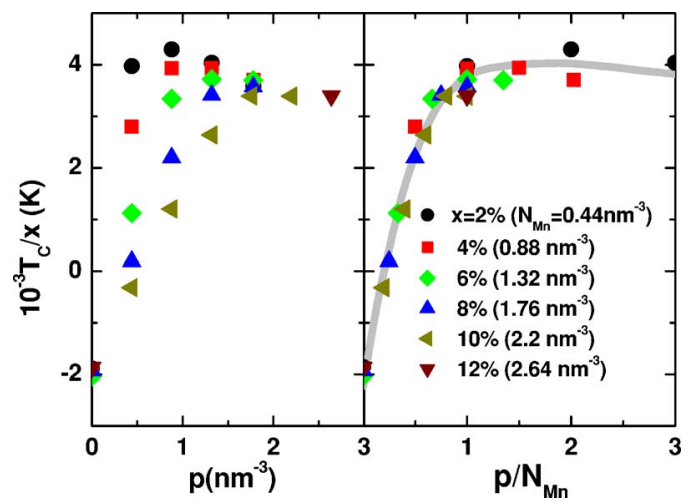

FIG. 2. (Color online) $T_{c}$ calculations within the microscopic TBA/CPA model: $T_{c}$ vs hole density (left panel), $T_{c}$ vs number of holes per $\mathrm{Mn}_{\mathrm{Ga}}$ (right panel). The overall theoretical $T_{c}$ trend is highlighted in gray to facilitate comparison with experimental data discussed in Sec. III C.

the appropriate exchange splitting of the Mn $d$ states. Local changes of the crystal potential at $\mathrm{Mn}_{\mathrm{Ga}}$, represented by shifted atomic levels, are estimated using Ref. 48. Longrange tails of the impurity potentials, which become less important with increasing level of doping, are neglected. (Note, that the Thomas-Fermi screening length is only $3-5 \AA$ for typical carrier densities, ${ }^{49}$ i.e., comparable to the lattice constant.) Also lattice relaxation effects are neglected within the CPA.

In our TBA/CPA calculations, hole density is varied independently of $\mathrm{Mn}_{\mathrm{Ga}}$ doping by adding nonmagnetic donors ( $\mathrm{Si}$ or $\mathrm{Se}$ ) or acceptors $(\mathrm{C}$ or $\mathrm{Be})$. The resulting valence-band splitting is almost independent of the density of these nonmagnetic impurities at fixed $N_{\mathrm{Mn}}$, which indicates that quasiparticle broadening due to positional disorder has only a weak effect on the strength of the kinetic-exchange coupling. We intentionally did not use $\mathrm{Mn}_{\mathrm{I}}$ donors in these calculations to avoid mixing of the (arguably) most important effect of this defect which is moment compensation. This is discussed separately in Sec. II E.

The TBA/CPA Curie temperatures are obtained using the compatibility of the model with the Weiss mean-field theory. The strength of the $\mathrm{Mn}_{\mathrm{Ga}}-\mathrm{Mn}_{\mathrm{Ga}}$ coupling is characterized by the energy cost of flipping one $\mathrm{Mn}_{\mathrm{Ga}}$ moment, which can be calculated for a given chemical composition. ${ }^{50}$ This effective field $H_{e f f}$ corresponds to $H_{M F}$ in the semiphenomenological kinetic-exchange model used in the previous section, i.e.,

$$
k_{B} T_{c}=\frac{S+1}{3} H_{e f f}
$$

In Fig. 2 we plot the mean-field TBA/CPA transition temperatures as a function of hole densities for several $\mathrm{Mn}_{\mathrm{Ga}}$ concentrations. Since the typical $T_{c}$ 's here are similar to those in Fig. 1 we can identify, based on the comparison between the two figures, the main physical origins of the deviations from the $T_{c} \sim x p^{1 / 3}$ trend. Closed circles in the left panel of Fig. 2, which correspond to a relatively low local $\mathrm{Mn}_{\mathrm{Ga}}$ moment concentration $(x=2 \%)$ and hole densities 
ranging up to $p=4 N_{\mathrm{Mn}}$, show the expected supression of $T_{c}$ at large $p$. The effect of superexchange in the opposite limit is clearly seen when inspecting, e.g., the $x=10 \%$ data for $p<1 \mathrm{~nm}^{-3}$. The mean-field TBA/CPA Curie temperature is largely suppressed here or even negative, meaning that the ferromagnetic state becomes unstable due to the short-range antiferromagnetic coupling. Also, the inhomogeneity of the carrier distribution in the disordered mixed crystal may contribute to the steep decrease of $T_{c}$ with increasing compensation. Although the Curie temperatures in the left panel of Fig. 2 appear to depart strongly for the $T_{c} \sim x p^{1 / 3}$ dependence, the linearity with $x$ is almost fully recovered when $T_{c}$ is plotted as a function of the number of holes per $\mathrm{Mn}_{\mathrm{Ga}}$, $p / N_{\text {Mn }}$ (see right panel of Fig. 2). Note that for compensations $\left(1-p / N_{\mathrm{Mn}}\right)$ reaching $100 \%$ this property of the superexchange coupling is reminiscent of the behavior of (II,Mn)VI diluted magnetic semiconductors ${ }^{51}$ in which Mn acts as an isovalent magnetic impurity. The dependence on $p$ in $(\mathrm{Ga}, \mathrm{Mn}) \mathrm{As}$ is expected to become very weak, however, when reaching the uncompensated limit or when further increasing hole density by nonmagnetic acceptor co-doping.

\section{Collective Mn-moment fluctuations}

The potential importance of correlated Mn-moment fluctuations on $T_{c}$ in $(\mathrm{Ga}, \mathrm{Mn})$ As can be illustrated by recalling, within a simple parabolic band model, the RKKY (or Friedel) oscillations effect which occurs as a consequence of the $2 k_{F}$ anomaly in the wave vector dependent susceptibility of the hole system. ${ }^{14,42}$ In this picture, the sign of the indirect kinetic-exchange $\mathrm{Mn}_{\mathrm{Ga}}-\mathrm{Mn}_{\mathrm{Ga}}$ coupling fluctuates as $\cos \left(2 k_{F} d\right)$, where $d$ is the distance between $\mathrm{Mn}_{\mathrm{Ga}}$ moments, and its amplitude decays as $d^{3}$. We can estimate the average $\mathrm{Mn}_{\mathrm{Ga}}-\mathrm{Mn}_{\mathrm{Ga}}$ separation in a $(\mathrm{Ga}, \mathrm{Mn})$ As random alloy as $\bar{d}=2\left(3 / 4 \pi N_{\mathrm{Mn}}\right)^{1 / 3}$. If the spin-orbit interaction and bandwarping are neglected, the top of the valence band is formed by six degenerate parabolic bands with $k_{F}=\left(\pi^{2} p\right)^{1 / 3}$. For uncompensated $(\mathrm{Ga}, \mathrm{Mn})$ As systems $\left(p=N_{\mathrm{Mn}}\right)$, we then obtain $\cos \left(2 k_{F} \bar{d}\right) \approx-1$ which means that the role of these fluctuations cannot be generally discarded. In realistic valence bands, as we see below, the fluctuations are suppressed due to nonparabolic and anisotropic dispersions of the heavy-and light-hole bands and due to the strong spin-orbit coupling.

On a more quantitative level, we can establish the range of reliability and estimate corrections to the mean-field theory in $(\mathrm{Ga}, \mathrm{Mn})$ As by accounting for the suppression of the Curie temperature within quantum theory of longwavelength spin waves in the semiphenomenological virtualcrystal model. We note that a qualitatively similar picture is obtained using Monte Carlo simulations which treat Mnmoments as classical variables and account for positional disorder. ${ }^{14,43,52}$ Isotropic ferromagnets have spin-wave Goldstone collective modes whose energies vanish at long wavelengths,

$$
\Omega_{k}=D k^{2}+\mathcal{O}\left(k^{4}\right),
$$

where $k$ is the wave vector of the mode. Spin-orbit coupling breaks rotational symmetry and leads to a finite gap. Accord- ing to numerical studies, ${ }^{33}$ this gap is small however, much smaller than $k_{B} T_{c}$, for example, and plays a role in magnetic fluctuations only at very low temperatures. Spin-wave excitations reduce the total spin by one, at an energy cost that is, at least at long wavelengths, much smaller than the meanfield value, $H_{M F}$. The importance of these correlated spin excitations, neglected by mean-field theory, can be judged by evaluating an approximate $T_{c}$ bound based on the following argument which uses a Debye-like model for the magnetic excitation spectrum. When spin-wave interactions are neglected, the magnetization vanishes at the temperature where the number of excited spin waves equals the total spin of the ground state:

$$
N_{\mathrm{Mn}} S=\frac{1}{2 \pi^{2}} \int_{0}^{k_{D}} d k k^{2} n\left(\Omega_{k}\right),
$$

where $n\left(\Omega_{k}\right)$ is the Bose occupation number and the Debye cutoff, $k_{D}=\left(6 \pi^{2} N_{\mathrm{Mn}}\right)^{1 / 3}$. It follows that the ferromagnetic transition temperature cannot exceed

$$
k_{B} T_{c}=\frac{2 S+1}{6} k_{D}^{2} D\left(T_{c}\right) .
$$

In applying this formula to estimate $T_{c}$ we have approximated the temperature dependence of the spin stiffness by

$$
D(T)=D_{0}\langle S\rangle(T) / S,
$$

where $D_{0}$ is the zero-temperature stiffness, ${ }^{33,53}$ and $\langle S\rangle(T)$ is the mean-field Mn polarization ${ }^{26}$ at temperature $T$. If the difference between $T_{c}$ obtained from the self-consistent solution of Eqs. (11) and (12) and the mean-field Curie temperature in Eq. (4) is large, the typical local valence-band carrier polarization will remain finite above the critical temperature and the ferromagnetism will disappear only because of the loss of long-range spatial order; for example, the usual circumstance for transition metal ferromagnetism.

In discussing corrections to mean-field-theory $T_{c}$ estimates, we compare spin-stiffness results obtained with the simple two-band and realistic six-band models. Details on the formalism used to calculate $D_{0}$ can be found in Refs. 33 and 54 . We find that the zero-temperature spin stiffness is always much larger in the six-band model. For $(\mathrm{Ga}, \mathrm{Mn}) \mathrm{As}$, the two-band model underestimates $D_{0}$ by a factor of $\sim 10-30$ over the range of hole densities considered. Furthermore, the trend is different: in the two-band model the stiffness decreases with increasing density, while for the sixband description the initial increase is followed by a saturation. Even in the limit of low carrier concentrations, it is not only the (heavy-hole) mass of the lowest band which is important for the spin stiffness. In the realistic band model, heavy-holes have their spin and orbital angular momenta aligned approximately along the direction of the Bloch wave vector. Exchange interactions with Mn spins mix the heavy holes with more dispersive light holes. The calculations show that heavy-light mixing is responsible for the relatively large spin stiffnesses. Crudely, the large mass heavy-hole band dominates the spin susceptibility and enables local magnetic order at high temperatures, while the dispersive light-hole band dominates the spin stiffness and enables 
long-range magnetic order. The multiband character of the semiconductor valence band plays an important role in the ferromagnetism of these materials.

Closed symbols in Fig. 1 summarize critical temperature estimates that include both the Stoner enhancement of $T_{c}$ and the suppression due to spin-wave fluctuations. The data were calculated using the six-band Kohn-Luttinger model for hole densities up to one hole per $\mathrm{Mn}_{\mathrm{Ga}}$ and $\mathrm{Mn}_{\mathrm{Ga}}$ concentrations $x=5 \%, 8 \%$, and $10 \%$. Given the qualitative nature of these $T_{c}$ estimates we can conclude that $T_{c}$ will remain roughly proportional to $x$ even at large dopings. The suppression of $T_{c}$ due to spin waves increases with increasing hole density relative to the local moment concentration, resulting in saturation of the critical temperature with increasing $p$ at about $50 \%$ compensation.

\section{E. $M n_{G a}$ and $M n_{I}$ partial concentrations}

In the previous sections we have considered $\mathrm{Mn}$ to occupy only the Ga substitutional positions and found that $T_{c}$ should increase linearly with the concentration of $\mathrm{Mn}_{\mathrm{Ga}}$ local moments. In real $(\mathrm{Ga}, \mathrm{Mn})$ As materials a fraction of $\mathrm{Mn}$ is incorporated during the growth in interstitial positions. These donor impurities are likely to form pairs with $\mathrm{Mn}_{\mathrm{Ga}}$ acceptors in as-grown systems with approximately zero net moment, $8,37,38$ resulting in an effective free local-moment doping $x_{e f f}=x_{s}-x_{i}$. Here $x_{s}$ and $x_{i}$ are partial concentrations of substitutional and interstitial Mn, respectively. Although $\mathrm{Mn}_{\mathrm{I}}$ can be removed by low-temperature annealing, $x_{\text {eff }}$ will remain smaller than the total nominal Mn doping. The $\mathrm{Mn}_{\mathrm{Ga}}$ doping efficiency is, therefore, one of the key parameters that may limit maximum $T_{c}$ achieved in $(\mathrm{Ga}, \mathrm{Mn})$ As epilayers.

In this section, we calculate cohesion energy $E_{c}\left(x_{s}, x_{i}\right)$ as a function of the partial concentrations $x_{s}$ and $x_{i}$ and we use it to determine the dependence of $x_{s}$ and $x_{i}$ on the total Mn doping in as-grown materials. We define $E_{c}\left(x_{s}, x_{i}\right)$ as a difference of the crystal energy per unit cell and a properly weighted sum of energies of isolated constituent atoms. The cohesion energy is not very sensitive to the details of the electronic structure and can be calculated with a reasonable accuracy, for example, by using the microscopic TBA model. Note that the growth kinetics calculations ${ }^{55}$ identified adsorption pathways for $\mathrm{Mn}_{\mathrm{I}}$ formation in $(\mathrm{Ga}, \mathrm{Mn}) \mathrm{As}$ epilayers. Our equilibrium consideration provides, as seen in Sec. III B, a very good estimate for the fraction of Mn impurities incorporated in interstitial positions.

The partial Mn concentrations $x_{s}$ and $x_{i}$ can be obtained by minimizing $E_{c}\left(x_{s}, x_{i}\right)$ at fixed Mn concentration $x=x_{s}+x_{i}$, with respect to either $x_{s}$ or $x_{i}$. Formally, the condition for a dynamical equilibrium between the two positions of Mn has a form

$$
\frac{\partial E_{c}\left(x_{s}, x_{i}\right)}{\partial x_{s}}-\frac{\partial E_{c}\left(x_{s}, x_{i}\right)}{\partial x_{i}}=0
$$

It was recently shown ${ }^{56}$ that the partial derivatives of the cohesion energy $E_{c}\left(x_{s}, x_{i}\right)$ with respect to $x_{s}$ and $x_{i}$ represent formation energies $F_{s}$ and $F_{i}$ of $\mathrm{Mn}_{\mathrm{Ga}}$ and $\mathrm{Mn}_{\mathrm{I}}$ impurities, respectively, assuming that the atomic reservoir is formed by

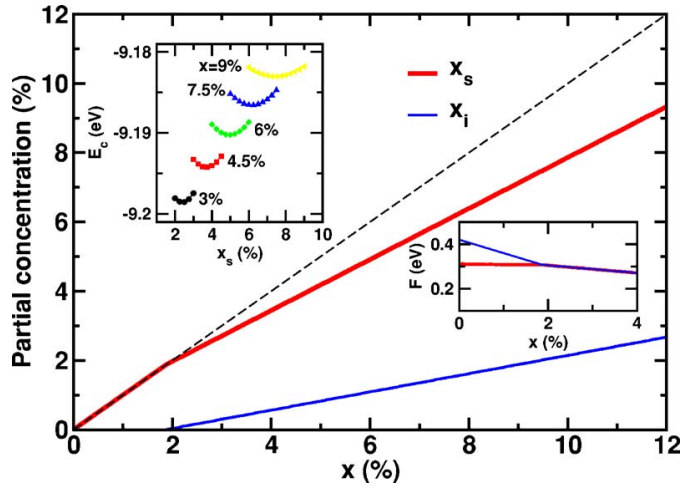

FIG. 3. (Color online) Main panel: Theoretical equilibrium partial concentrations of substitutional $\mathrm{Mn}_{\mathrm{Ga}}$ (red thick line) and interstitial $\mathrm{Mn}_{\mathrm{I}}$ (blue thin line) impurities. Right inset: Formation energies of $\mathrm{Mn}_{\mathrm{Ga}}$ and $\mathrm{Mn}_{\mathrm{I}}$ as a function of total Mn concentration. Left inset: Cohesion energy as a function of substitutional $\mathrm{Mn}_{\mathrm{Ga}}$ concentration at several fixed total Mn concentrations.

neutral isolated atoms. The equilibrium distribution of $\mathrm{Mn}_{\mathrm{Ga}}$ and $\mathrm{Mn}_{\mathrm{I}}$ is therefore reached when

$$
F_{s}\left(x_{s}, x_{i}\right)=F_{i}\left(x_{s}, x_{i}\right)
$$

as expected also from the growth point of view. Partial concentrations $x_{s, i}$ of Mn can be obtained by solving Eq. (13) together with the condition $0 \leqslant x_{s, i} \leqslant x, x_{s}+x_{i}=x$.

The left inset of Fig. 3 summarizes the compositional dependence of the cohesion energy in $(\mathrm{Ga}, \mathrm{Mn})$ As with both $\mathrm{Mn}_{\mathrm{Ga}}$ and $\mathrm{Mn}_{\mathrm{I}}$ impurities. We consider several values of $x$ and plot $E_{c}\left(x_{s}, x-x_{s}\right)$ vs $x_{s}$. Although the changes of the cohesion energy due to the incorporation of $\mathrm{Mn}$ are small, a systematic linear shift of the minimum of $E_{c}$ with increasing $x$ is clearly visible. Correspondingly, the partial concentration of $x_{s, i}$ is expected to increase with increasing $x$. For $x$ $>1.5 \%$ we obtain $x_{s} \approx 0.8 x$ and $x_{i} \approx 0.2 x$, in good agreement with the density-functional results. ${ }^{34}$

The linear relations between $x_{s}, x_{i}$, and $x$ reflect the fact that the difference of the formation energies of $\mathrm{Mn}_{\mathrm{Ga}}$ and $\mathrm{Mn}_{\mathrm{I}}$ impurities (see right inset of Fig. 3) can be, up to $x$ $=10 \%$, approximated by a linear function of $x_{s}$ and $x_{i}$,

$$
\Delta\left(x_{s}, x_{i}\right) \equiv F_{s}\left(x_{s}, x_{i}\right)-F_{i}\left(x_{s}, x_{i}\right) \approx-0.1+5.9 x_{s}-15.1 x_{i}(\mathrm{eV}) .
$$

This relation allows us to interpret the theoretical distribution of Mn atoms between substitutional and interstitial sites. For $x<1.5 \%, \mathrm{Mn}_{\mathrm{Ga}}$ has a lower formation energy than $\mathrm{Mn}_{\mathrm{I}}$ and $\mathrm{Mn}$ atoms tend to occupy substitutional positions. At $x$ $\approx 1.5 \%, \Delta\left(x_{s}, x_{i}\right)$ approaches zero and both $\mathrm{Mn}_{\mathrm{Ga}}$ and $\mathrm{Mn}_{\mathrm{I}}$ are formed with a similar probability, as shown in Fig. 3.

We note that both $\mathrm{Mn}_{\mathrm{Ga}}$ and $\mathrm{Mn}_{\mathrm{I}}$ positions remain metastable in the whole concentration range shown in Fig. 3 and that our results correspond to the as-grown rather than to the annealed materials. During the growth, the formation energies [namely, $\left.\Delta\left(x_{s}, x_{i}\right)\right]$ control incorporation of $\mathrm{Mn}$ atoms assuming that the total amount of $\mathrm{Mn}$ in the material is related to a sufficiently high chemical potential in the $\mathrm{Mn}$ source. The annealing processes, on the other hand, do not 
depend on formation energies but rather on energy barriers surrounding individual metastable positions of $\mathrm{Mn}$ in the lattice. The barriers are larger for $\mathrm{Mn}_{\mathrm{Ga}}$ (Refs. 38 and 55) so that the post-grown low-temperature annealing can be used to remove $\mathrm{Mn}_{\mathrm{I}}$ without changing the number of $\mathrm{Mn}_{\mathrm{Ga}}$ significantly.

\section{F. Hole density and Hall coefficient}

As discussed above, the level of compensation is one of the key parameters that determines Curie temperatures in $(\mathrm{Ga}, \mathrm{Mn})$ As. In this paper, as well as in a number of other experimental works, hole densities are obtained from Hall measurements. In order to estimate the uncertainty of this experimental technique we analyze in this section theoretical Hall factors, $r_{H}=\left(\rho_{x y}-\rho_{x y, 0}\right) /(B / e p)$, in ferromagnetic $(\mathrm{Ga}, \mathrm{Mn})$ As epilayers. Here $\rho_{x y, 0}$ is the Hall resistivity at field $B=0$ which can be nonzero due to the anomalous Hall effect.

Detailed microscopic calculations in nonmagnetic $p$-type GaAs with hole densities $p \sim 10^{17}-10^{20} \mathrm{~cm}^{-3}$ showed that $r_{H}$ can vary between 0.87 and 1.75 , depending on doping, scattering mechanisms, and on the level on which the complexity of the GaAs valence band is modeled ${ }^{57}$ Here we focus on estimating the effect on $r_{H}$ of the spin-splitting of the valence band and of the anomalous Hall term that is particularly large in ferromagnetic $(\mathrm{Ga}, \mathrm{Mn}) \mathrm{As}$.

The calculations are based on numerical evaluation of the Kubo formula at finite magnetic fields. We assume band- and wave-vector-independent quasiparticle lifetimes for simplicity. It is essential for our analysis to allow for both intraband and interband transitions. At zero magnetic field, the interband transitions between SO-coupled, spin-split bands give rise to the anomalous Hall effect (AHE), i.e., to a nonzero $\rho_{x y}$ that is proportional to the magnetization. ${ }^{58}$ On the other hand, the ordinary Hall resistance, which is proportional to $B$, arises, within the simple single-band model, from intraband transitions between adjacent Landau levels. The Kubo formula that includes both intraband and interband transitions allows us to capture simultaneously the anomalous and ordinary Hall effects in the complex $(\mathrm{Ga}, \mathrm{Mn}) \mathrm{As}$ valence bands.

Many of the qualitative aspects of the numerical data shown in Figs. 4 and 5 can be explained using a simple model of a conductor with two parabolic uncoupled bands. Note that the typical scattering rate in $(\mathrm{Ga}, \mathrm{Mn})$ As epilayers is $\hbar / \tau \sim 100 \mathrm{meV}$ and the cyclotron energy at $B=5 \mathrm{~T}$ is $\hbar \omega \sim 1 \mathrm{meV}$, i.e., the system is in the strong scattering limit, $\omega \tau \ll 1$. In this limit, the two band model gives resistivities:

$$
\begin{gathered}
\rho_{x x} \approx \frac{1}{\sigma_{x x, 1}+\sigma_{x x, 2}} \approx \frac{1}{\sigma_{0,1}+\sigma_{0,2}} \\
\rho_{x y} \approx-\frac{\sigma_{x y, 1}+\sigma_{x y, 2}}{\left(\sigma_{x x, 1}+\sigma_{x x, 2}\right)^{2}}=\frac{B}{e p_{1}} \frac{1+\frac{p_{2}}{p_{1}}\left(\frac{m_{1}^{*}}{m_{2}^{*}}\right)^{2}}{\left(1+\frac{p_{2}}{p_{1}} \frac{m_{1}^{*}}{m_{2}^{*}}\right)^{2}} \geqslant \frac{B}{e p},
\end{gathered}
$$

where the indices 1 and 2 correspond to the first and second band, respectively, the total density $p=p_{1}+p_{2}$, and the zero-

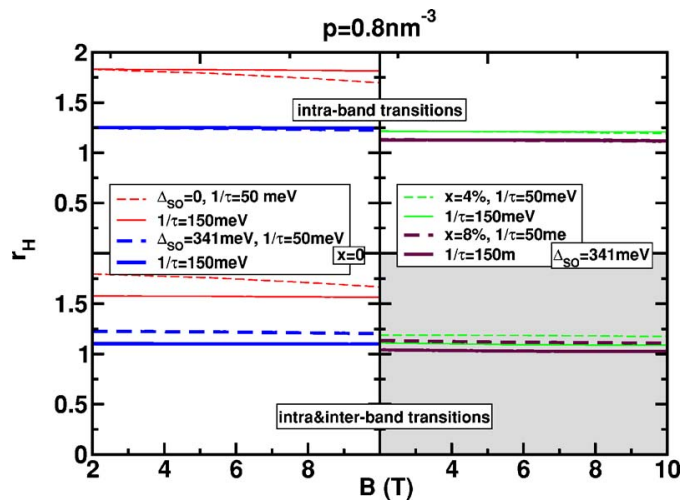

FIG. 4. (Color online) Theoretical Hall factors for $p=0.8 \mathrm{~nm}^{-3}$; $\hbar / \tau=50 \mathrm{meV}$ (dashed lines), $\hbar / \tau=150 \mathrm{meV}$ (solid lines). Top panels: only intraband transitions are taken into account. Bottom panels: intraband and interband transitions are taken into account. Left panels: GaAs $(x=0)$; zero SO coupling (thin lines), $\Delta_{S O}$ $=341 \mathrm{meV}$ (thick lines). Right panels: $(\mathrm{Ga}, \mathrm{Mn}) \mathrm{As}$ with $\mathrm{Mn}_{\mathrm{Ga}}$ concentration $4 \%$ (thin lines), $8 \%$ (thick lines). $\rho_{x y}=0$ in all panels except for the bottom left panel where $\rho_{x y}(B=0) \neq 0$ due to the anomalous Hall effect.

field conductivity $\sigma_{0}=e^{2} \tau p / m^{*}$. Equation (16) suggests that in the strong scattering limit the multiband nature of the hole states in $(\mathrm{Ga}, \mathrm{Mn})$ As should not result in a strong longitudinal magnetoresistance. This observation is consistent with the measured weak dependence of $\rho_{x x}$ on $B$ for magnetic fields at which magnetization in the $(\mathrm{Ga}, \mathrm{Mn}) \mathrm{As}$ ferromagnet is saturated. ${ }^{59}$

The simple two-band model also suggests that the Hall factor $r_{H}$ is larger than 1 in multiband systems with different dispersions of individual bands. Indeed, for uncoupled valence bands, i.e., when accounting for intraband transitions only, the numerical Hall factors in the top panels of Figs. 4 and 5 are larger than 1 and independent of $\tau$ as also suggested by Eq. (16). The suppression of $r_{H}$ when SO coupling is turned on, shown in the same graphs, results partly from depopulation of the angular momentum $j=\frac{1}{2}$ split-off bands. In addition to this "two-band model"-like effect, the interLandau-level matrix elements are reduced due to SO coupling since the spinor part of the eigenfunctions now varies

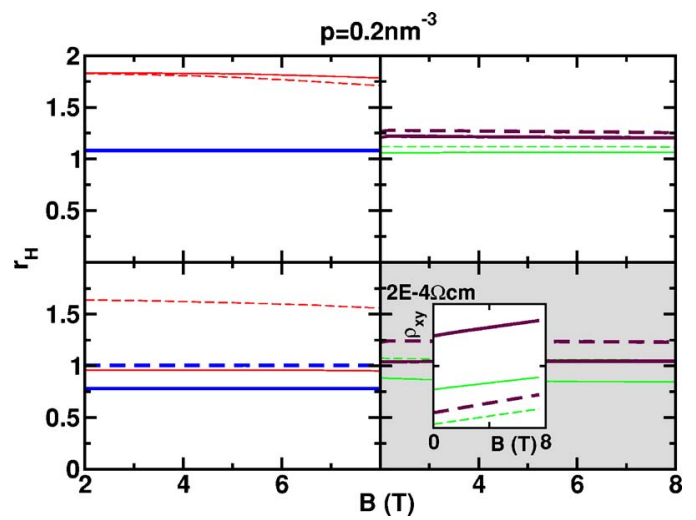

FIG. 5. (Color online) Theoretical Hall factors for $p=0.2 \mathrm{~nm}^{-3}$; same line coding as in Fig. 4. Inset: Theoretical Hall curves showing the anomalous Hall effect contribution at $B=0$. 
with the Landau-level index. In ferromagnetic $\mathrm{Ga}_{1-x} \mathrm{Mn}_{x} \mathrm{As}$ the bands are spin-split and higher bands depopulated as $x$ increases. In terms of $r_{H}$, this effect competes with the increase of the inter-Landau-level matrix elements since the spinors are now more closely aligned within a band due to the exchange field produced by the polarized Mn moments. Increasing $x$ can therefore lead to both decrease or increase of $r_{H}$ depending on other parameters of the $(\mathrm{Ga}, \mathrm{Mn}) \mathrm{As}$, such as the hole density (compare top right panels of Figs. 4 and 5).

The interband transitions result in a more single-band-like character of the system, i.e., $r_{H}$ is reduced, and the slope of the $\rho_{x y}(B)$ curve now depends more strongly on $\tau$. Although the anomalous and ordinary Hall effect contributions to $\rho_{x y}$ cannot be simply decoupled, the comparison of numerical data in the four panels and the inset in Fig. 5 confirms the usual assumption that the anomalous Hall effect produces a field-independent off-set proportional to magnetization and $\rho_{x x}^{2}$. The comparison also suggests that after subtracting $\rho_{x y}(B=0), r_{H}$ can be used to determine the hole density in $(\mathrm{Ga}, \mathrm{Mn})$ As with accuracy that is better than in nonmagnetic GaAs with comparable hole densities. For typical hole and Mn densities in experimental (Ga,Mn)As epilayers we estimate the error of the Hall measurement of $p$ to be within $\pm 20 \%$.

\section{EXPERIMENT}

\section{A. Measured Curie temperatures and hole densities}

A series of $(\mathrm{Ga}, \mathrm{Mn})$ As thin films with varying Mn content were grown by LT-MBE using $\mathrm{As}_{2}$. The layer structure is 25 or $50 \mathrm{~nm}(\mathrm{Ga}, \mathrm{Mn}) \mathrm{As} / 50-\mathrm{nm}$ low-temperature GaAs/100$\mathrm{nm}$ high-temperature $\left(580{ }^{\circ} \mathrm{C}\right) \mathrm{GaAs} / \mathrm{GaAs}(100)$ substrate. For a given $\mathrm{Mn}$ content, the growth temperature of the $(\mathrm{Ga}, \mathrm{Mn})$ As layer and the GaAs buffer is chosen in order to minimize As antisite densities while maintaining twodimensional growth and preventing phase segregation. We find that the growth temperature must be decreased as the Mn concentration is increased: for the lowest Mn content the growth temperature was $\sim 300{ }^{\circ} \mathrm{C}$, for the highest it was $\sim 180{ }^{\circ} \mathrm{C}$. Full details of the growth are presented elsewhere..$^{60,61}$

The Mn content was controlled by varying the $\mathrm{Mn} / \mathrm{Ga}$ incident flux ratio, measured in situ and calibrated using secondary-ion-mass spectroscopy (SIMS) measurements on $1 \mu \mathrm{m}$ thick $(\mathrm{Ga}, \mathrm{Mn})$ As films, grown under otherwise identical conditions to the samples considered here. A detailed comparison of the results of a number of different calibration techniques, presented in Ref. 62, allows us to assign an uncertainty of $\pm 10 \%$ to the quoted Mn concentrations. However, it should be noted that the SIMS measurements yield the total volume density of $\mathrm{Mn}$ in the (Ga,Mn)As films, and not the fraction of Ga substituted by Mn. This is important as it is expected that a fraction of the Mn will be incorporated on interstitial as well as substitutional sites. ${ }^{3}$ We define the Mn concentration $x$ as the total Mn volume density relative to the volume density of $\mathrm{Ga}$ in GaAs.

Hall bar structures, of width $200 \mu \mathrm{m}$ and length $1 \mathrm{~mm}$, were fabricated from the $(\mathrm{Ga}, \mathrm{Mn}) \mathrm{As}$ samples using photoli-

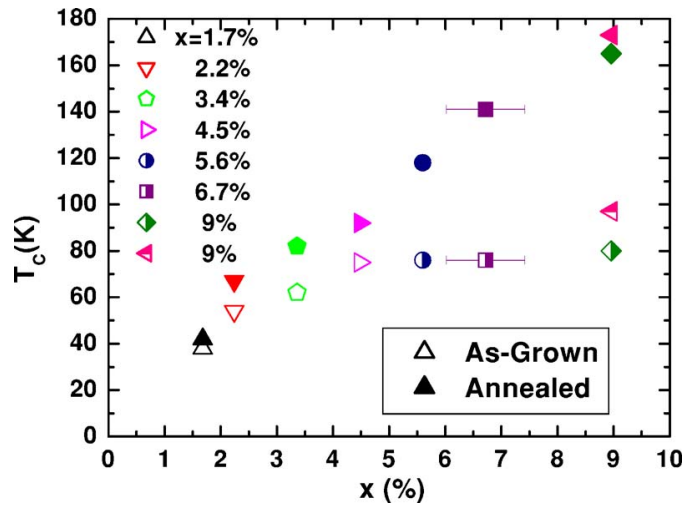

FIG. 6. (Color online) Experimental Curie temperature vs total $\mathrm{Mn}$ doping. $T_{c}$ is measured from the anomalous Hall effect, Mn doping by SIMS. Open symbols correspond to as-grown samples, half-open symbols to as-grown samples with large charge compensation, and filled symbols to annealed samples. For clarity, error bars are shown only for the $x=6.7 \%$ sample.

thography. Simultaneous magnetoresistance and Hall effect measurements were performed using standard low-frequency ac techniques, in order to extract both the Curie temperature $T_{c}$ and the hole density $p$, as detailed below. Magnetic fields of up to $\pm 0.7 \mathrm{~T}$ and $\pm 16.5 \mathrm{~T}$ were used to obtain $T_{c}$ and $p$, respectively. Following these measurements, the samples were annealed in air at $190{ }^{\circ} \mathrm{C}$. The electrical resistance was monitored during annealing, and the anneal was halted when this appeared to have reached a minimum (after typically 50 to $150 \mathrm{~h}$ ). The $T_{c}$ and $p$ were then remeasured.

Below $T_{c}$, the Hall resistance $R_{x y}$ in $(\mathrm{Ga}, \mathrm{Mn}) \mathrm{As}$ is dominated by the anomalous Hall effect, with $R_{x y} \sim R_{A} M_{z}$, where $M_{z}$ is the perpendicular component of the magnetization, and the coefficient $R_{A}$ is roughly proportional to the square of the resistivity, $\rho_{x x}$. Therefore, $R_{x y} / \rho_{x x}^{2}$ gives a direct measurement of $M_{z}$, which can be used to extract $T_{c}$ using Arrot plots. ${ }^{63}$ The value of $T_{c}$ obtained depends only weakly on the precise dependence of $R_{A}$ on $\rho_{x x}$ assumed, since $\rho_{x x}$ varies only slowly close to $T_{c}$, while $R_{x y}$ varies rapidly. We are therefore able to obtain $T_{c}$ within an accuracy of $\pm 1 \mathrm{~K}$ by this method. ${ }^{59}$

$T_{c}$ obtained for the $(\mathrm{Ga}, \mathrm{Mn})$ As Hall bar samples before and after annealing are shown vs $x$ in Fig. 6. It can be seen that the low-temperature annealing procedure results in a marked increase in $T_{c}$ as has been found previously. ${ }^{64}$ Increases of $T_{c}$ by more than a factor of 2 are possible. This effect becomes larger as the Mn concentration increases. Since the $T_{c}$ enhancement is associated with out-diffusion and passivation of interstitial $\mathrm{Mn},{ }^{8}$ this indicates that as the incident Mn flux is increased, an increasing fraction is incorporated on interstitial sites, as predicted in Sec. II E.

To obtain hole densities from $R_{x y}$, it is necessary to separate the small normal Hall term from the much larger anomalous Hall term. Measurements were performed at $0.3 \mathrm{~K}$ and in magnetic fields above $10 \mathrm{~T}$, i.e., under conditions where the normal Hall term gives the dominant field-dependent contribution to $R_{x y}$. Then, the measured $R_{x y}$ was fitted to $\left(\alpha \rho^{2}+r_{H} B\right)$, where $\rho_{x x}$ and $B$ are the measured resistivity and magnetic field, and $\alpha$ and $r_{H}$ are fit parameters. Finally, the 


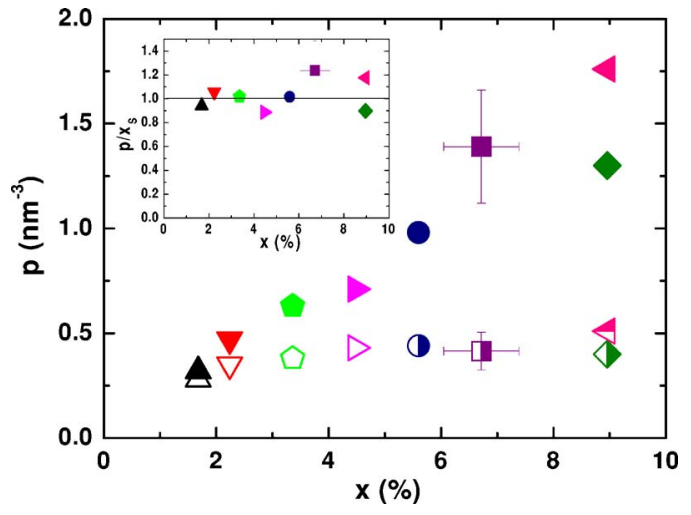

FIG. 7. (Color online) Experimental hole density vs total Mn doping. Hole density is measured by ordinary Hall effect. Same symbol coding is used as in Fig. 6.

hole density is obtained from $r_{H}=1 /($ pew $)$, where $w$ is the $(\mathrm{Ga}, \mathrm{Mn})$ As layer thickness. From the detailed calculations described in Sec. II F we can ascribe an uncertainty of $\pm 20 \%$ to the values of $p$ obtained using this method. The measured $p$ for the $(\mathrm{Ga}, \mathrm{Mn})$ As Hall bar samples before and after annealing are shown vs $x$ in Fig. 7. We see that annealing greatly increases $p$ for large $x$. Data in the inset of Fig. 7, discussed in detail in Sec. III B, show that within error the samples are uncompensated after annealing.

\section{B. Substitutional and interstitial Mn}

From the measured hole density $p$ before and after annealing, and the total Mn density $x$, values can be obtained for the density of incorporated Mn occupying acceptor substitutional and double donor interstitial lattice sites, $x_{s}$ and $x_{i}$. These are obtained using the following assumptions: (i) the only contribution to the total Mn density determined by SIMS are from substitutional and interstitial $\mathrm{Mn}$, i.e., $x=x_{s}$ $+x_{i}$; (ii) the only source of compensation in the $(\mathrm{Ga}, \mathrm{Mn}) \mathrm{As}$ films are the interstitial $\mathrm{Mn}$, which are double donors, i.e., $p=4 / a_{l c}^{3}\left(x_{s}-2 x_{i}\right)$; (iii) the low-temperature annealing procedure affects only $x_{i}$, and not $x_{s}$. The values of $x_{s}$ and $x_{i}$ in the unannealed films obtained under these assumptions are shown in Fig. 8. We find a remarkably good agreement between experiment and the theoretical TBA/CPA data from Sec. II E and $a b$ initio results. ${ }^{34}$ As a consistency check, we show in the inset of Fig. 7 the ratio of hole density to substitutional $\mathrm{Mn}_{\mathrm{Ga}}$ density after annealing, as obtained under the above assumptions. Within the experimental error we obtain one hole per substitutional $\mathrm{Mn}_{\mathrm{Ga}}$ after annealing, that is, there is no significant compensation in the annealed (Ga,Mn)As films. This justifies our neglect of additional compensating defects such as $\mathrm{As}_{\mathrm{Ga}}$ in determining $x_{s}$ and $x_{i}$.

\section{C. $T_{c}$ vs $\mathrm{Mn}_{\mathrm{Ga}}$, effective $\mathrm{Mn}_{\mathrm{Ga}}$, and hole densities}

Since we obtain reasonably accurate values for $T_{c}$, hole densities and the partial $\mathrm{Mn}_{\mathrm{Ga}}$ and $\mathrm{Mn}_{\mathrm{I}}$ concentrations for the set of samples considered here, we now attempt to assess on the basis of the experimental data the key factors determin-

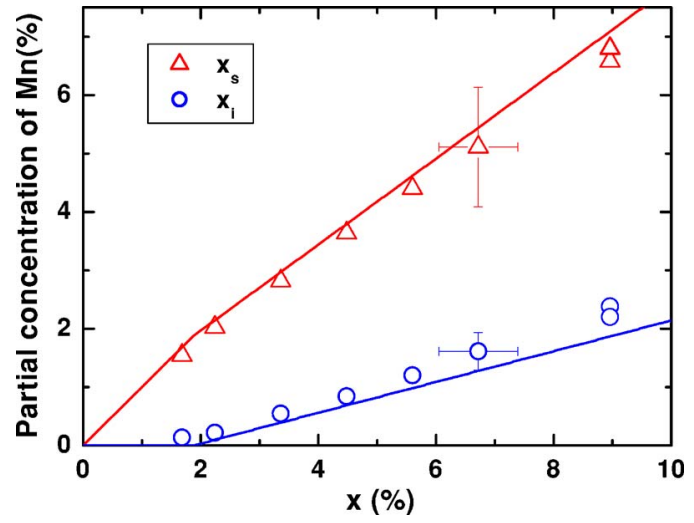

FIG. 8. (Color online) Experimental partial concentrations of $\mathrm{Mn}_{\mathrm{Ga}}$ (triangles) and $\mathrm{Mn}_{\mathrm{I}}$ (circles) in as-grown samples. Data show no saturation of $\mathrm{Mn}_{\mathrm{Ga}}$ with increasing total $\mathrm{Mn}$ doping consistent with theory expectation (solid lines).

ing $T_{c}$ and to compare the experimental results with the broad predictions of theory.

In Fig. $6 T_{c}$ was plotted against total Mn concentration. Before annealing the $T_{c}$ values of samples with high compensation (samples with large compensation are indicated as half filled symbols in this and subsequent figures) do not increase significantly with increasing total Mn density but a steady increase is recovered after annealing. In Fig. $9 T_{c}$ is plotted against the substitutional $\mathrm{Mn}_{\mathrm{Ga}}$ concentration. The form of Figs. 6 and 9 are broadly similar despite the different $x$ axes. We expect, however, and will assume in the following discussion that any $\mathrm{Mn}_{\mathrm{I}}$ donor present is attracted to a $\mathrm{Mn}_{\mathrm{Ga}}$ acceptor and that the pair couples antiferromagnetically. ${ }^{38}$ Then the effective uncompensated moment density will be $x_{e f f}=x_{s}-x_{i}$. Plotting $T_{c}$ against $x_{e f f}$ in Fig. 10 reveals that for all the low compensation samples $T_{c}$ increases approximately linearly with $x_{\text {eff }}$ but that as compensation, $\left(1-p a_{l c}^{3} / 4 x_{e f f}\right)$, increases above $\sim 40 \%$ the measured $T_{c}$ values fall increasingly far below this linear trend.

If $T_{c}$ is plotted against hole density, as is done in the inset of Fig. 11, it is found to increase monotonically. However, this is primarily due to the increase in hole density with $x_{\text {eff }}$. The main plot in Fig. 11 shows that $T_{c} / x_{\text {eff }}$ is almost inde-

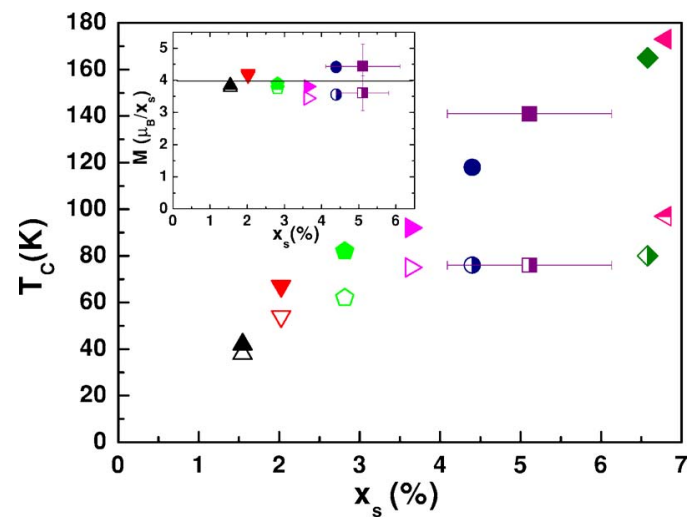

FIG. 9. (Color online) Experimental $T_{c}$ vs $\mathrm{Mn}_{\mathrm{Ga}}$ concentration, $x_{s}$ (see text for definition of $x_{s}$ ). Magnetization per $x_{s}$ is shown in the inset. 


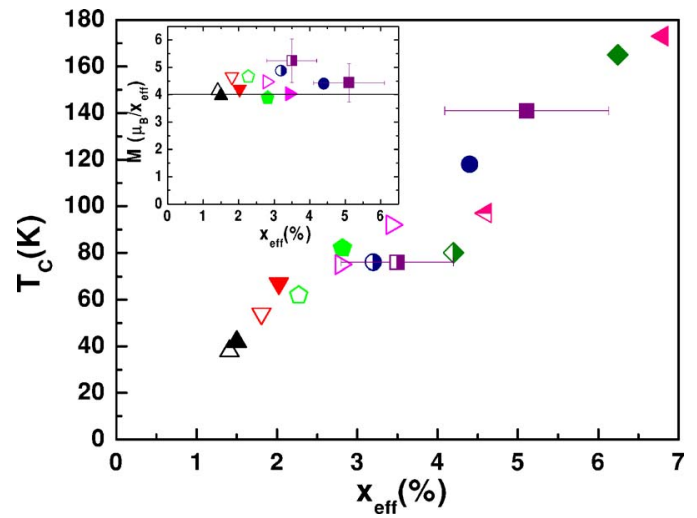

FIG. 10. (Color online) Experimental $T_{c}$ vs effective $\mathrm{Mn}_{\mathrm{Ga}}$ concentration, $x_{e f f}$ (see text for definition of $x_{e f f}$ ). Magnetization per $x_{e f f}$ is shown in the inset.

pendent of hole density except for the case of the high compensation samples which clearly stand out as showing different behavior.

To compare with the predictions of Sec. II, we finally plot $T_{c} / x_{\text {eff }}$ against $p / N_{\text {Mn }}^{e f f}$ in Fig. 12, where $N_{\text {Mn }}^{e f f}=4 x_{\text {eff }} / a_{l c}^{3}$. We emphasize here that a precise comparison between theory and experiment is not meaningful as the modeling techniques do not allow us to capture the complexities of ferromagnetic states in $(\mathrm{Ga}, \mathrm{Mn}) \mathrm{As}$ on a fully quantitative level, as explained in the Introduction. On the other hand, all experimental points in Fig. 12 show a common $T_{c}$ trend which is consistent with the theoretical models discussed in Sec. II and the magnitudes of the experimental and calculated $T_{c} / x_{e f f}$ are also comparable. Further confirmation of the theoretical picture is seen from the very weak experimental dependence of $T_{c} / x_{\text {eff }}$ on $p / N_{\mathrm{Mn}}^{\text {eff }}$ for low compensation and the relatively rapid fall of $T_{c} / x_{e f f}$ with decreasing $p / M_{\mathrm{Mn}}^{e f f}$ for compensations of $\sim 40 \%$ or larger.

As a consistency check for considering $x_{e f f}$ as the density of local $\mathrm{Mn}_{\mathrm{Ga}}$ moments participating in the ordered ferromagnetic state, magnetization data are shown in insets of Figs. 9 and 10. Magnetizations were determined by superconducting quantum interference device magnetometry, at a sample temperature of $5 \mathrm{~K}$, and using an external field of

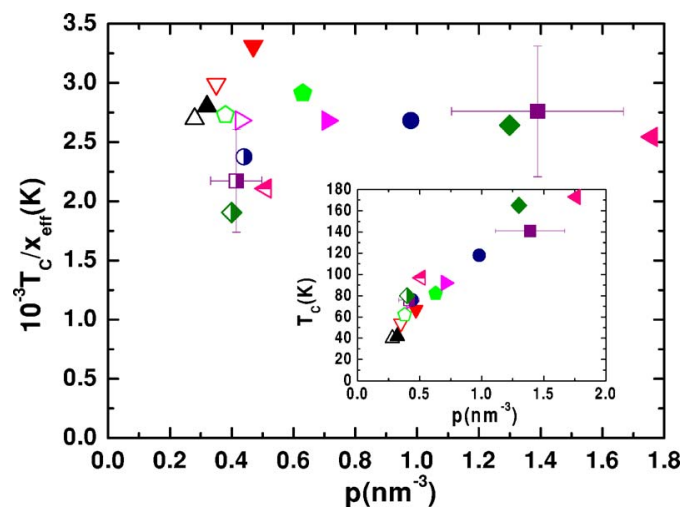

FIG. 11. (Color online) Experimental $T_{c} / x_{e f f}$ vs hole density. $T_{c} / x_{e f f}$ is nearly independent of hole density except in highly compensated samples. Inset: $T_{c}$ vs hole density.

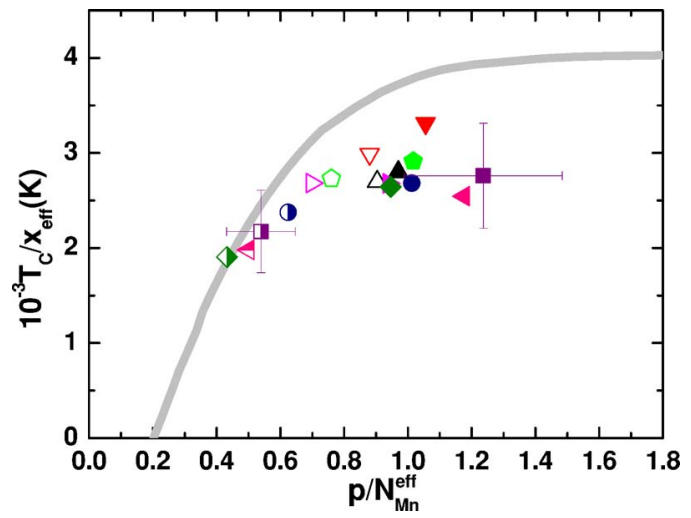

FIG. 12. (Color online) Experimental $T_{c} / x_{\text {eff }}$ vs hole density relative to effective concentration of $\mathrm{Mn}_{\mathrm{Ga}}$ moments. Deviation from linear dependence on $x_{e f f}$ are seen only for high compensations $\left(1-p a_{l c}^{3} / 4 x_{e f f}>40 \%\right)$ in agreement with theory. For weakly compensated samples $T_{c}$ shows no signs of saturation with increasing $x_{\text {eff }}$. Theoretical (gray) $T_{c}$ trend from Fig. 2 is plotted for comparison.

$0.3 \mathrm{~T}$ to overcome in-plane anisotropy fields. The charge and moment compensation after annealing is not significant for our samples and the moment per $x_{s}$ or $x_{\text {eff }}$ is around $\sim 4 \mu_{B}-4.5 \mu_{B}$. This corresponds within the error bars to the $5 \mu_{B}$ contribution of the $\mathrm{S}=\frac{5}{2}$ local $\mathrm{Mn}_{\mathrm{Ga}}$ moment and $\sim(-0.25)-(-0.5) \mu_{B}$ contribution of the antiferromagnetically coupled valence-band hole ${ }^{25}$ in collinear (Ga,Mn)As ferromagnets. In the inset of Fig. 9 we see that the measured moment per $x_{s}$ are all below $4 \mu_{B}$ for the compensated samples. Including the effects of the $\mathrm{Mn}_{\mathrm{I}}-\mathrm{Mn}_{\mathrm{Ga}}$ antiferromagnetic coupling by considering the moment per $x_{e f f}$ reveals again values around $4.5 \mu_{B}$. Our conclusion, therefore, is that if we assume no significant frustration in our samples and account for the antiferromagnetic $\mathrm{Mn}_{\mathrm{I}}-\mathrm{Mn}_{\mathrm{Ga}}$ coupling, our extensive set of $T_{c}$, hole density, Mn density, and magnetization data brings up a clear common picture of $T_{c}$ trends in the 16 different (Ga,Mn)As ferromagnetic semiconductors we have studied, that is consistent with the theory predictions summarized in Sec. II.

\section{DISCUSSION}

The preceding considerations of the factors determining $T_{c}$ in (Ga,Mn)As lead us to conclude that there are no fundamental physics barriers to achieving room temperature ferromagnetism in this system. Experimental results for $T_{c}$ in samples in which compensating defects other than interstitial Mn have been reduced to very low levels have been shown to be in good agreement with theoretical expectations. Moment compensation by interstitial $\mathrm{Mn}_{\mathrm{I}}$ impurities becomes increasingly important as the concentration of total $\mathrm{Mn}$ is increased. However, for the range of total Mn concentrations considered experimentally we find that the level of substitutional $\mathrm{Mn}_{\mathrm{Ga}}$ continues to increase with $x$. Furthermore lowtemperature post-growth annealing is found to effectively remove $\mathrm{Mn}_{\mathrm{I}}$ in thin film samples even at large $x$, leading to material which within experimental error is both charge and 
moment uncompensated. Most importantly for samples in which the charge compensation is less than $\sim 40 \%$, we find theoretically and experimentally that $T_{C}$ increases approximately linearly with effective concentration $x_{\text {eff }}$ of $\mathrm{Mn}_{\mathrm{Ga}}$, whose moments are not compensated by near-neighbor $\mathrm{Mn}_{\mathrm{I}}$ impurities. We have not observed any signs of saturation in this trend in the studied (Ga,Mn)As diluted magnetic semiconductors. It should be noted that our maximum $x_{\text {eff }}$ is only $4.6 \%$ in the as grown sample and $6.8 \%$ after annealing for a total Mn concentration $x=9 \%$; hence the modest $T_{c}$ 's observed so far. Achieving $T_{c}$ values close to room temperature in $(\mathrm{Ga}, \mathrm{Mn}) \mathrm{As}$, which we expect to occur for $x_{\text {eff }} \approx 10 \%$ is essentially a technological issue, albeit a very challenging one. In the remaining paragraphs of this section we discuss these challenges in more detail.

Low-temperature MBE growth is used to achieve levels of $\mathrm{Mn}$ incorporation in $(\mathrm{Ga}, \mathrm{Mn}) \mathrm{As}$ far in excess of the equilibrium solubility level. When growing $(\mathrm{Ga}, \mathrm{Mn})$ As with $\mathrm{Mn}$ concentrations of several percent it is known that the Mn tends to accumulate on the surface ${ }^{60,61}$ in a similar way to all high vapor pressure dopants in GaAs and to the higher vapor pressure species, e.g. In in $\mathrm{In}_{x} \mathrm{Ga}_{1-x}$ As. For homogenous $\mathrm{Mn}$ incorporation during continuous growth, a surface Mn concentration is required that is higher than the bulk concentration. For a given Mn concentration this density gradient is temperature dependent, increasing with increasing temperature. This leads to an upper temperature limit for successful growth when the Mn surface concentration approaches a significant proportion of a monolayer, after which point surface clustering of Mn occurs, frustrating the growth. ${ }^{60,61}$ Furthermore, higher Mn fluxes require lower growth temperatures.

The pursuit of higher Curie temperatures has driven growth efforts to very low temperatures compared with conventional MBE of GaAs. In this regime $\left(\sim 200-250{ }^{\circ} \mathrm{C}\right)$ significant levels of compensating defects such as $\mathrm{As}_{\mathrm{Ga}}$ and vacancies usually occur in GaAs. ${ }^{65}$ The density of $\mathrm{As}_{\mathrm{Ga}}$ defects can be reduced by close to stoichiometric growth with $\mathrm{As}_{2},{ }^{66}$ requiring very precise control over the As flux.

Apart from precise control over the stoichiometry and the attendant requirement for flux stability, a major technical difficulty arises from the measurement and control of the growth temperature. In order to measure substrate temperature, most MBE machines in use today employ a thermocouple heated by radiation from the substrate or substrate holder. At normal growth temperatures $\left(\sim 580{ }^{\circ} \mathrm{C}\right)$ the radiant flux from the substrate is high and the relationship between substrate and thermocouple is repeatable with a short time constant, allowing for good temperature stability and control. At low temperatures, however, the radiant flux between the substrate and thermocouple is low, leading to a heightened sensitivity to local conditions such as holder emissivity, radiant heat from the metal sources, shutter transients etc., and also long time constants. This significantly increases the error in the temperature measurement as well as the likelihood of temperature spikes and drift as shutters are opened and growth proceeds. In MBE, optical pyrometers are ubiquitous as secondary temperature calibration devices but most cannot read accurately at these low temperatures and in many common configurations suffer from potential inaccuracies due to reflection off the Knudsen cells if used during growth.

It is desirable to grow at as high temperature as possible for a given Mn flux, while maintaining two-dimensional (2D) growth and avoiding Mn clustering. However, with such large errors and potential temperature drift, growers tend to err toward lower than ideal temperatures in order to sustain the growth. To explore fully the parameter space, effort should be directed toward improving the control of both metal fluxes and substrate temperature. This will maximize the chances of increasing the doping towards the $10 \%$ $\mathrm{Mn}_{\mathrm{Ga}}$, required for room temperature ferromagnetism. The increases in $T_{c}$ achieved in the last few years lead us to believe that higher transition temperatures will be obtained using conventional MBE. Growth interrupt strategies such as migration enhanced epitaxy $y^{67}$ may have advantages over conventional MBE for the incorporation of higher levels of substitutional Mn, however, they will be especially sensitive to poor temperature stability and shutter transients and so they will require even more precise temperature control.

\section{CONCLUSION}

Based on the broad agreement between theoretical and experimental Curie temperature trends in $(\mathrm{Ga}, \mathrm{Mn}) \mathrm{As}$ with Mn concentrations larger than $1.5 \%$ we can outline the following strategies for achieving room temperature ferromagnetism in this semiconductor.

(i) $T_{c}$ increases linearly with the concentration $x_{\text {eff }}$ of local $\mathrm{Mn}_{\mathrm{Ga}}$ moments participating in the ordered ferromagnetic state. Room temperature ferromagnetism should be achieved at $x_{\text {eff }} \approx 10 \%$. Interstitial $\mathrm{Mn}_{\mathrm{I}}$ impurities reduce the number of these ordered $\mathrm{Mn}_{\mathrm{Ga}}$ moments due to the strong antiferromagnetic $\mathrm{Mn}_{\mathrm{Ga}}-\mathrm{Mn}_{\mathrm{I}}$ near-neighbor coupling. $\mathrm{Mn}_{\mathrm{I}}$, however, can be efficiently removed by post-growth annealing.

(ii) Equilibrium considerations, confirmed experimentally in samples with $\mathrm{Mn}_{\mathrm{Ga}}$ concentrations up to $6.8 \%$, suggest that there is no fundamental physics barrier for increasing $\mathrm{Mn}_{\mathrm{Ga}}$ concentration to and beyond $10 \%$. A very precise control over the growth temperature and stoichiometry is, however, required for maintaining the $2 \mathrm{D}$ growth mode of the high quality, uniform $(\mathrm{Ga}, \mathrm{Mn}) \mathrm{As}$ materials.

(iii) Ferromagnetic coupling between the ordered local $\mathrm{Mn}_{\mathrm{Ga}}$ moments is mediated by itinerant holes. For charge compensations $\left(1-p a_{l c}^{3} / 4 x_{e f f}\right)>40 \%$, the Curie temperature falls down with decreasing $p$. At compensations smaller than $\sim 40 \%$, however, $T_{c}$ is almost independent of the hole density. A modest charge compensation is, therefore, not an important limiting factor in the search of high Curie temperature ferromagnetic semiconductors based on $(\mathrm{Ga}, \mathrm{Mn}) \mathrm{As}$ and may be desirable to maximize the possibilities for doping and gate control of ferromagnetism.

\section{ACKNOWLEDGMENTS}

We acknowledge support from the Grant Agency of the Czech Republic through Grant No. 202/05/0575 from the Academy of Sciences of the Czech Republic through Insti- 
tutional Support No. AV0Z10100521, from the Ministry of Education of the Czech Republic through the Center for Fundamental Research LC510, from the EU FENIKS Project No. EC:G5RD-CT-2001-00535, the support from the UK
EPSRC through Grant No. GR/S81407/01, from the Welch Foundation, the Department of Energy under Grant No. DEFG03-02ER45958, and from Deutsche Forschungsgemeinschaft through Grant No. SFB 491.
${ }^{1}$ H. Ohno, Science 281, 951 (1998).

${ }^{2}$ S. J. Potashnik, K. C. Ku, R. Mahendiran, S. H. Chun, R. F. Wang, N. Samarth, and P. Schiffer, Phys. Rev. B 66, 012408 (2002).

${ }^{3}$ K. M. Yu, W. Walukiewicz, T. Wojtowicz, I. Kuryliszyn, X. Liu, Y. Sasaki, and J. K. Furdyna, Phys. Rev. B 65, 201303(R) (2002).

${ }^{4}$ K. M. Yu, W. Walukiewicz, T. Wojtowicz, W. L. Lim, X. Liu, U. Bindley, M. Dobrowolska, and J. K. Furdyna, Phys. Rev. B 68, 041308(R) (2003).

${ }^{5}$ K. Edmonds, K. Wang, R. Campion, A. Neumann, N. Farley, B. Gallagher, and C. Foxon, Appl. Phys. Lett. 81, 4991 (2002).

${ }^{6}$ D. Chiba, K. Takamura, F. Matsukura, and H. Ohno, Appl. Phys. Lett. 82, 3020 (2003).

${ }^{7}$ K. C. Ku, S. J. Potashnik, R. F. Wang, M. J. Seong, E. JohnstonHalperin, R. C. Meyers, S. H. Chun, A. Mascarenhas, A. C. Gossard, D. D. Awschalom, P. Schiffer, and N. Samarth, Appl. Phys. Lett. 82, 2302 (2003).

${ }^{8}$ K. Edmonds, P. Boguslawski, K. Wang, R. Campion, N. Farley, B. Gallagher, C. Foxon, M. Sawicki, T. Dietl, M. Nardelli, and J. Bernholc, Phys. Rev. Lett. 92, 037201 (2004).

${ }^{9}$ K. Wang, R. Campion, K. Edmonds, M. Sawicki, T. Dietl, C. Foxon, and B. Gallagher, AIP Conf. Proc. 772, 333 (2005).

${ }^{10}$ M. B. Stone, K. C. Ku, S. J. Potashnik, B. L. Sheu, N. Samarth, and P. Schiffer, Appl. Phys. Lett. 83, 4568 (2003).

${ }^{11}$ T. Jungwirth, W. A. Atkinson, B. H. Lee, and A. H. MacDonald, Phys. Rev. B 59, 9818 (1999).

${ }^{12}$ T. Dietl, H. Ohno, F. Matsukura, J. Cibert, and D. Ferrand, Science 287, 1019 (2000).

${ }^{13}$ T. Jungwirth, J. König, J. Sinova, J. Kučera, and A. H. MacDonald, Phys. Rev. B 66, 012402 (2002).

${ }^{14}$ L. Brey and G. Gómez-Santos, Phys. Rev. B 68, 115206 (2003).

${ }^{15}$ S. Das Sarma, E. H. Hwang, and D. J. Priour, Jr., Phys. Rev. B 70, 161203(R) (2004).

${ }^{16}$ L. M. Sandratskii and P. Bruno, Phys. Rev. B 66, 134435 (2002).

${ }^{17}$ K. Sato, P. H. Dederichs, and H. Katayama-Yoshida, Europhys. Lett. 61, 403 (2003).

${ }^{18}$ L. M. Sandratskii, P. Bruno, and J. Kudrnovský, Phys. Rev. B 69, 195203 (2004).

${ }^{19}$ S. Hilbert and W. Nolting, Phys. Rev. B 71, 113204 (2005).

${ }^{20}$ T. Jungwirth, J. Mašek, J. Sinova, and A. H. MacDonald, Phys. Rev. B 68, 161202(R) (2003).

${ }^{21}$ C. Timm, J. Phys.: Condens. Matter 15, R1865 (2003).

${ }^{22}$ I. Vurgaftman, J. Meyer, and L. Ram-Mohan, J. Appl. Phys. 89, 5815 (2001).

${ }^{23}$ J. Okabayashi, A. Kimura, O. Rader, T. Mizokawa, A. Fujimori, T. Hayashi, and M. Tanaka, Phys. Rev. B 58, R4211 (1998).

${ }^{24}$ T. Omiya, F. Matsukura, T. Dietl, Y. Ohno, T. Sakon, M. Motokawa, and H. Ohno, Physica E (Amsterdam) 7, 976 (2000).

${ }^{25}$ T. Dietl, H. Ohno, and F. Matsukura, Phys. Rev. B 63, 195205 (2001).

${ }^{26}$ M. Abolfath, T. Jungwirth, J. Brum, and A. H. MacDonald, Phys.
Rev. B 63, 054418 (2001)

${ }^{27}$ M. Sawicki, F. Matsukura, A. Idziaszek, T. Dietl, G. M. Schott, C. Ruester, C. Gould, G. Karczewski, G. Schmidt, and L. M. Molenkamp, Phys. Rev. B 70, 245325 (2004).

${ }^{28}$ M. Sawicki, K.-Y. Wang, K. W. Edmonds, R. Campion, C. Staddon, N. Farley, C. Foxon, E. Papis, E. Kaminska, A. Piotrowska, T. Dietl, and B. L. Gallagher, Phys. Rev. B 71, 121302(R) (2005).

${ }^{29}$ T. Jungwirth, J. Sinova, K. Wang, K. W. Edmonds, R. Campion, B. Gallagher, C. Foxon, Q. Niu, and A. H. MacDonald, Appl. Phys. Lett. 83, 320 (2003).

${ }^{30}$ H. X. Tang, R. K. Kawakami, D. D. Awschalom, and M. L. Roukes, Phys. Rev. Lett. 90, 107201 (2003).

${ }^{31}$ C. Gould, C. Rüster, T. Jungwirth, E. Girgis, G. M. Schott, R. Giraud, K. Brunner, G. Schmidt, and L. W. Molenkamp, Phys. Rev. Lett. 93, 117203 (2004).

${ }^{32}$ A. Giddings, M. Khalid, T. Jungwirth, J. Wunderlich, S. Yasin, R. Campion, K. Edmonds, J. Sinova, K. Ito, K. Y. Wang, D. Williams, B. L. Gallagher, and C. T. Foxton, Phys. Rev. Lett. 94, 127202 (2005).

${ }^{33}$ J. König, T. Jungwirth, and A. H. MacDonald, Phys. Rev. B 64, 184423 (2001).

${ }^{34}$ J. Mašek, I. Turek, J. Kudrnovský, F. Máca, and V. Drchal, Acta Phys. Pol. A 105, 637 (2004).

${ }^{35}$ F. Máca and J. Mašek, Phys. Rev. B 65, 235209 (2002).

${ }^{36}$ J. Kudrnovský, I. Turek, V. Drchal, J. Mašek, F. Máca, and P. Weinberger, J. Supercond. 16, 119 (2003).

${ }^{37}$ J. Blinowski and P. Kacman, Phys. Rev. B 67, 121204(R) (2003).

${ }^{38}$ J. Mašek and F. Máca, Phys. Rev. B 69, 165212 (2003).

${ }^{39}$ D. E. Bliss, W. Walukiewicz, J. W. Ager, III, E. E. Haller, K. T. Chan, and S. Tanigawa, J. Appl. Phys. 71, 1699 (1992).

${ }^{40}$ R. P. Campion, K. W. Edmonds, L. X. Zhao, K. Y. Wang, C. T. Foxon, B. L. Gallagher, and C. R. Staddon, J. Cryst. Growth 251, 311 (2003).

${ }^{41}$ J. Kudrnovský, I. Turek, V. Drchal, F. Máca, P. Weinberger, and P. Bruno, Phys. Rev. B 69, 115208 (2004).

${ }^{42}$ T. Dietl, A. Haury, and Y. M. d'Aubigne, Phys. Rev. B 55, R3347 (1997).

${ }^{43}$ J. König, J. Schliemann, T. Jungwirth, and A. MacDonald, in Electronic Structure and Magnetism of Complex Materials, edited by D. Singh and D. Papaconstantopoulos (Springer-Verlag, Berlin, 2003); cond-mat/0111314 (unpublished).

${ }^{44}$ C. Timm and A. H. MacDonald, Phys. Rev. B 71, 155206 (2005).

${ }^{45}$ G. A. Fiete, G. Zaránd, B. Jankó, P. Redliński, and C. P. Moca, Phys. Rev. B 71, 115202 (2005).

${ }^{46}$ P. Mahadevan and A. Zunger, Appl. Phys. Lett. 85, 2860 (2004).

${ }^{47}$ D. N. Talwar and C. S. Ting, Phys. Rev. B 25, 2660 (1982).

${ }^{48}$ W. Harrison, Electronic Structure and the Properties of Solid (Freeman, San Francisco, 1980).

${ }^{49}$ T. Jungwirth, M. Abolfath, J. Sinova, J. Kučera, and A. MacDonald, Appl. Phys. Lett. 81, 4029 (2002).

${ }^{50}$ J. Mašek, Solid State Commun. 78, 351 (1991). 
${ }^{51}$ J. K. Furdyna, J. Appl. Phys. 64, R29 (1988).

${ }^{52}$ J. Schliemann, J. König, and A. H. MacDonald, Phys. Rev. B 64, 165201 (2001).

${ }^{53}$ J. Schliemann, J. König, H. Lin, and A. MacDonald, Appl. Phys. Lett. 78, 1550 (2001).

${ }^{54}$ J. König, H. H. Lin, and A. H. MacDonald, Phys. Rev. Lett. 84, 5628 (2000).

${ }^{55}$ S. C. Erwin and A. G. Petukhov, Phys. Rev. Lett. 89, 227201 (2002).

${ }^{56}$ J. Mašek, I. Turek, V. Drchal, J. Kudrnovský, and F. Máca, Acta Phys. Pol. A 102, 673 (2002); cond-mat/0302176 (unpublished).

${ }^{57}$ B. W. Kim and A. Majerfeld, Prog. Oceanogr. 17, 17 (1995).

${ }^{58}$ J. Sinova, T. Jungwirth, and J. Černe, Int. J. Mod. Phys. B 18, 1083 (2004).

${ }^{59}$ K. W. Edmonds, K. Y. Wang, R. P. Campion, A. C. Neumann, C. T. Foxon, B. L. Gallagher, and P. C. Main, Appl. Phys. Lett. 81, 3010 (2002).

${ }^{60}$ R. Campion, K. Edmonds, L. Zhao, K. Wang, C. Foxon, B. Gal- lagher, and C. Staddon, J. Cryst. Growth 247, 42 (2003).

${ }^{61}$ C. T. Foxon, R. P. Campion, K. W. Edmonds, L. Zhao, K. Wang, N. R. S. Farley, C. R. Staddon, and B. L. Gallagher, J. Mater. Sci. 15, 727 (2004).

${ }^{62}$ L. X. Zhao, R. P. Campion, P. F. Fewster, R. W. Martin, B. Y. Ber, A. P. Kovarsky, C. R. Staddon, K. Y. Wang, K. W. Edmonds, C. T. Foxon, and B. L. Gallagher, Semicond. Sci. Technol. 20, 369 (2005).

${ }^{63}$ F. Matsukura, H. Ohno, A. Shen, and Y. Sugawara, Phys. Rev. B 57, R2037 (1998).

${ }^{64}$ T. Hayashi, Y. Hashimoto, S. Katsumoto, and Y. Iye, Appl. Phys. Lett. 78, 1691 (2001).

${ }^{65}$ D. Hurle, J. Appl. Phys. 85, 6957 (1999).

${ }^{66}$ M. Missous and K. E. Singer, Appl. Phys. Lett. 50, 694 (1987).

${ }^{67}$ J. Sadowski, R. Mathieu, P. Svedlindh, M. Karlsteen, J. Kanski, L. Ilver, H. Åsklund, K. Swiatek, J. Z. DomagaIa, J. BakMisiuk, and D. Maude, Physica E (Amsterdam) 10, 181 (2001). 